\title{
Various Image Processing Attacks for Image Watermarking in the Wavelet Domain Using Singular Value Decomposition and Discrete Cosine Transform
}

\author{
Riyajuddin*, Arikera Padmanabha Reddy \\ Department of Studies in Mathematics, Vijayanagara Sri Krishnadevaraya University, Ballari 583105, Karnataka, India
}

Corresponding Author Email: riyazuddin.k108@gmail.com

https://doi.org/10.18280/rces.080205

Received: 15 May 2021

Accepted: 21 June 2021

\section{Keywords:}

digital image watermarking, Haar wavelet, truncated singular value decomposition, peak signal to noise ratio, normalized correlation, mean square error

\begin{abstract}
The dispersal of digital media due to the fast evolution of networked multimedia systems has created an essential need for copyright prompting technologies that can protect multimedia objects such as text, images, audio and videos from copyright ownership. This paper proposes digital image watermarking algorithm for copyright protection based on discrete wavelet transform, discrete cosine transform and singular value decomposition. In this method a watermark is embedded into the low frequency sub-band of a host image, after subjecting the watermarked image to various attacks like Gaussian noise, rotation sharpening, noise and pepper salt and speckle noise etc., we extract the originally inserted watermark images from LL sub-band by Truncated singular value decomposition and compare them on the basis of their mean square error, peak signal to noise ratio and normalized correlation values. Experimental results are provided to illustrate that the proposed scheme is the robustness of the technique on wide set of attacks.
\end{abstract}

\section{INTRODUCTION}

The internet is an excellent dispensation system for digital media as it is expensive eliminates warehousing and stock, delivery is almost instantaneous and it quickly becomes clear that people want to copy videos, pictures and music. However, content owners also see a high risk of unauthorized use. To tackle with copyright issues, digital watermark is then introduced to solve this problem. Digital watermarking is a process of inserting watermark information into cover image. Watermark is the copyright information which protects digital data from the illegal replication and distribution.

Watermarking schemes can be divided into two categories according to the embedding domain: spatial-domain and frequency-domain [1]. Compared to spatial-domain watermarking techniques, frequency-domain techniques proved to be more effective with respect to achieving the imperceptibility and robustness requirements of watermarking algorithms [2]. Frequency-domain transform include the Discrete Wavelet Transform (DWT), the Discrete Cosine Transform (DCT) and Discrete Fourier Transform (DFT). However DWT has been used in digital image watermarking eternally due to its excellent spatial localization and multiresolution characteristics, which are identical to the theoretical models of the human visual system [3,4]. The main advantage of wavelet transform method is their superior robustness and imperceptibility requirements of digital watermarking algorithms.

Several watermarking algorithms have used evolutionary algorithms to solve optimization problem in digital image watermarking and many image watermarking techniques combining these three transform DWT, DCT and Singular Value Decomposition (SVD) method have been proposed [5$17]$.

Golshan and Mohammadi [16] proposed DCT-DWT-SVD based intelligent algorithm to make improvements in imperceptibility and robustness under several attacks and different images. Mei et al. [18] proposed a digital image watermarking algorithm based on DWT and DCT method. In embedding process, the host image is decomposed into three level discrete wavelet transform and the watermark image is embedded only in high frequency band information of DWT image. Lai and Tsai [19] proposed an algorithm for image watermarking scheme based on DWT and SVD. In the embedding process, the host image is decomposed into four sub-bands and SVD is applied to horizontal $(\mathrm{H})$ and vertical (V) sub-bands diagonally. After that the singular values of $\mathrm{H}$ and $\mathrm{V}$ sub-band are modified with the watermark image and then apply SVD to them, respectively.

This paper familiarizes an algorithm of digital watermarking based on DWT, DCT and SVD technique to embed watermark image into the cover image and the proposed method has been extensively tested with various kinds of attacks such as rotation attack, salt and pepper attack, Gaussian noise attack, sharpening and speckle noise attack.

The rest of the paper is organized as follows. In section 2, we introduce the concepts DWT, DCT and SVD briefly. In section 3, proposed algorithm steps in detail and image quality and similarity parameters in section 4 . Section 5 presents the experimental results to demonstrate the performance of the proposed scheme. Concluding remarks given in section 6 .

\section{BACKGROUND OF MATHEMATICAL PRELIMINARIES}

The DWT, DCT and SVD transforms have been widely used in many digital watermarking applications. In this section we introduce these transforms briefly and outline their relatedness to the implementation of digital watermarking. 


\subsection{Discrete wavelet transform}

In mathematics, the Haar wavelet is a sequence of rescaled "square-shaped" functions which together produces a wavelet family or basis [20]. The Haar wavelet was proposed in 1909 by Alfred Haar. These Haar functions used to give an example of an orthonormal system for the family of square-integrable functions on the unit interval $[0,1]$. The Haar wavelet's mother wavelet function $\psi(t)$ can be described as

$$
\psi(t)=\left\{\begin{array}{cl}
1, & 0 \leq t<1 / 2 \\
-1 & 1 / 2 \leq t<1 \\
0 & \text { otherwise }
\end{array}\right.
$$

and its scaling function $\phi(t)$ can be defined as

$$
\phi(t)=\left\{\begin{array}{ll}
1, & 0 \leq t<1 \\
0 & \text { otherwise }
\end{array} .\right.
$$

Discrete wavelet transform is a mathematical tool for decomposing an image in hierarchically [21]. The multiresolution representation of the image by hierarchical process in a wavelet domain is most important for scalable transmission and successive decoding of the watermark [22]. It is used in many applications of signal processing such as compression, internet communications compression, video compression, object recognition and numerical analysis. The discrete wavelet transform divides the input image into four non-overlapping multiresolution sub-bands LL, LH, HL and HH. The sub-band LL represents the coarse-scale DWT coefficients while the sub-bands $\mathrm{LH}, \mathrm{HL}$ and $\mathrm{HH}$ represent the fine-scale of DWT coefficients. Discrete Haar wavelet transform is applied effectively in applications such as watermarking as well as image compression methods can benefit from a good model of the human visual system (HVS) because it provides a simple and computationally efficient approach for analyzing the inside aspects of an image or signal [23]. Due to its excellent spatial localization and multiresolution properties, the discrete wavelet transform is very applicable to identify the areas in the cover image where a watermark can be inserted effectively.

\subsection{Discrete cosine transform}

Discrete cosine transform is a finite sum of cosine functions oscillating and varying with different frequencies. The DCT, first proposed by Nasir Ahmed in 1972, is a widely used transformation technique in signal processing and data compression. The DCT is similar to the discrete Fourier transform for which it transforms a signal or image from the spatial domain to the frequency domain.

The discrete cosine transform is a technique for converting a signal into elementary frequency components [24]. It represents each pixel of an image as a sum of sinusoids of varying magnitudes and frequencies. For an input image $\mathrm{X}$ of size MXN, the DCT coefficients for the transformed output image $\mathrm{Y}$ are computed according to Eq. (3) shown below. $\mathrm{X}(\mathrm{m}, \mathrm{n})$ is the intensity of the pixel in row $\mathrm{m}$ and column $\mathrm{n}$ of the image $\mathrm{X}$ and $\mathrm{Y}(\mathrm{u}, \mathrm{v})$ is the DCT coefficient in row $\mathrm{u}$ and column $\mathrm{v}$ of the DCT matrix.

$$
\begin{aligned}
& \mathrm{Y}(\mathrm{u}, \mathrm{v})=\alpha_{\mathrm{u}} \alpha_{\mathrm{v}} \sum_{\mathrm{m}=0}^{\mathrm{M}-1} \sum_{\mathrm{n}=0}^{\mathrm{N}-1} \mathrm{X}(\mathrm{m}, \mathrm{n}) \cos \left(\frac{\pi(2 \mathrm{~m}+1) \mathrm{u}}{2 \mathrm{M}}\right) \\
& \cos \left(\frac{\pi(2 \mathrm{n}+1) \mathrm{v}}{2 \mathrm{~N}}\right), 0 \leq \mathrm{u} \leq \mathrm{M}-1 \text { and } 0 \leq \mathrm{v} \leq \mathrm{N}-1
\end{aligned}
$$

where, $\alpha_{\mathrm{u}}=\left\{\begin{array}{ll}\frac{1}{\sqrt{\mathrm{M}}}, & \mathrm{u}=0 \\ \sqrt{\frac{2}{\mathrm{M}}}, & 1 \leq \mathrm{u} \leq \mathrm{M}-1\end{array}\right.$ and $\alpha_{\mathrm{v}}=\left\{\begin{array}{ll}\frac{1}{\sqrt{\mathrm{N}}}, & \mathrm{v}=0 \\ \sqrt{\frac{2}{\mathrm{~N}}}, & 1 \leq \mathrm{v} \leq \mathrm{N}-1\end{array}\right.$.

\subsection{Singular value decomposition}

Every real matrix $A$ of size MX N can be decomposed into a product of three matrices of the form

$$
A=U S V^{T}
$$

where, the $U$ and $V$ are real unitary matrices such that $U U^{T}=I$, $V V^{T}=I$ and $S$ is called diagonal matrix holding the singular values of $A$ which are obtained by taking the square root of the eigen values of $A A^{T}$ and $A^{T} A$. The columns of $U$ are called the left singular vectors of $A$ and columns of $V$ are called right singular vectors of $A$ [25].

The above decomposition can also be expressed as

$$
\begin{gathered}
A=\sum_{i=1}^{r} u_{i} \sigma_{i} v_{i}^{T} \\
A=u_{1} \sigma_{1} v_{1}^{T}+u_{2} \sigma_{2} v_{2}^{T}+\cdots+u_{r} \sigma_{r} v_{r}^{T}
\end{gathered}
$$

where, $\mathrm{r}$ is the rank of the matrix $A . u_{i}$ and $v_{i}$ are called $i^{\text {th }}$ left and right singular vectors of $U$ and $V$ respectively. Also, $\sigma_{i}$ 's are singular values appearing in non-increasing order along the main diagonal of $S$.

The approximate truncated expansion of $A$ can be expressed as

$$
B=\sum_{i=1}^{k} u_{i} \sigma_{i} v_{i}^{T}
$$

where, $1 \leq k \leq \operatorname{rank}(B, t)$ called truncated value and $\operatorname{rank}(B, t)$ is the number of singular values of $B$ that are greater than tolerence (t). Here the two sums in Eq. (5) and Eq. (7) are not exactly same because $k$ truncate the number of singular values of $B$ that are greater than $\mathrm{t}$ [26].

SVD in digital watermarking gives the spectral information of both host and watermark images which helps to embed watermark information into the cover image.

\section{PROPOSED METHOD}

The proposed algorithm is a combined DWT, DCT and SVD based process which increases the robustness without much degradation of image quality against the image processing attacks. The algorithm has two parts, watermark embedding and watermark extraction as given below

\section{ALGORITHM:}

\section{Watermark embedding algorithm:}

Input: Gray scale host image I and original watemark image W

Output: $W_{E} \approx W$

Step 1. First of all we decompose the cover image into four sub-bands. In this paper, we use one level Haar transformation for decomposition of cover image I into four sub-bands. 


$$
[L L, H L, L H, H H]=d w t(\mathrm{I}) .
$$

Step 2. After performing DWT, we perform DCT to LL subband of cover image.

$$
D_{C}=\operatorname{dct}(L L) \text {. }
$$

Step 3. In the same way, we apply DCT to watermark image.

$$
D_{W}=\operatorname{dct}(W) .
$$

Step 4. Apply SVD to both DCT coefficients of LL sub-band of a cover image and DCT coefficients of watermark image. Suppose $D_{C}$ be the DCT coefficients of LL sub-band of a cover image and $D_{W}$ be the DCT coefficients of watermark image then,

$$
\begin{aligned}
& \operatorname{SVD}\left(D_{C}\right)=U_{C} S_{C} V_{C}{ }^{T} \\
& \operatorname{SVD}\left(D_{W}\right)=U_{W} S_{W} V_{W}{ }^{T} .
\end{aligned}
$$

Step 5. Now we add singular values of DCT coefficients of cover image and scaling factor multiplied by singular values of DCT coefficients of watermark image

$$
S_{\alpha}=S_{C}+\alpha \cdot S_{W}
$$

Step 6. Again apply SVD to $S_{\alpha}$

$$
\operatorname{SVD}\left(S_{\alpha}\right)=U^{*} S^{*} V^{* T}
$$

Step 7. Truncate the singular values of $S^{*}$ with the singular vectors of $U_{C}, V_{C}$ and obtain the modified DCT coefficients

$$
X_{k 1}=\sum_{i=1}^{k 1} u_{c i} s_{i}^{*} v_{c i}^{T}, 1 \leq k 1 \leq \operatorname{rank}\left(S^{*}, t\right) .
$$

where, $u_{c}, v_{c}$ are left and right singular vectors of $U_{C}$ and $V_{C}$ Also, $k 1$ is number of truncated singular values that are greater than tolerence $(\mathrm{t})$.

Step 8. Apply inverse discrete cosine transform to modified DCT coefficients $\left(X_{k 1}\right)$

$$
W_{\text {Idct }}=\operatorname{Idct}\left(X_{k 1}\right) \text {. }
$$

Step 9. Finally, obtain the watermarked image by applying inverse discrete wavelet transform to $W_{\text {Idct }}$

$$
W_{d}=I d w t\left(W_{\text {Idct }}, H L, L H, H H, \text { 'haar }^{\prime}\right) .
$$

We used 'haar' as a wavelet filter which works decomposing a image into four non-overlapping multiresolution sub-bands $L L, H L, L H$ and $H H$ called DWT coefficients in a frequency domain.

\section{Watermark extraction algorithm:}

Step 1. Apply one-level Haar wavelet transform decompose watermarked image $W_{d}$ (possibly distorted due to various kinds of attacks) into four sub-bands

$$
[L L 1, H L 1, L H 1, H H 1]=d w t\left(W_{d}\right) .
$$

Here $L L 1, H L 1, L H 1$ and $H H 1$ are Haar wavelet coefficients in a frequency domain obtained by applying DWT to the watermarked image $W_{d}$.

Step 2. We compute the DCT coefficients of LL1 sub-band of watermarked image by applying discrete cosine transform to LL1 sub-band

$$
D_{W}^{*}=\operatorname{dct}(L L 1) \text {. }
$$

Step 3. Compute singular value decomposition of DCT coefficients of LL1 sub-band of watermarked image

$$
\operatorname{SVD}\left(D_{W}^{*}\right)=U_{W}^{*} S_{W}^{*} V^{* T} \text {. }
$$

Step 4. Extract the singular values by using the formula and compute its SVD

$$
\begin{gathered}
S=\frac{S_{W}^{*}-S_{C}}{\alpha}, \\
S V D(S)=U_{S} S_{S} V_{S}^{T} .
\end{gathered}
$$

Step 5. Rearranging $S_{S}$ with original singular vector matrices $U_{W}, V_{W}$ of watermark image

$$
U_{W} S_{S} V_{W}^{T}=X
$$

Step 6. Truncate singular values of $S_{S}$ with the singular vectors of $U_{W}, V_{W}$

$$
X_{k 2}=\sum_{i=1}^{k 2} u_{w i} s_{s i} v_{w i}^{T} \text { and } 1 \leq k 2 \leq \operatorname{rank}\left(S_{s}, t\right)
$$

Step 7. Finally, extract watermark image $W_{E}$ by applying inverse discrete cosine transform to $X_{k 2}$

$$
W_{E}=\operatorname{Idct}\left(X_{k 2}\right) \text {. }
$$

\section{PERFORMANCE MEASURES}

The performance of the watermarking algorithm can be evaluated on the basis of its robustness and imperceptibility. Peak signal to noise ratio (PSNR) is widely used to measure imperceptibility between the original image and watermarked image. A larger peak signal to noise ratio (PSNR) indicates that the watermarked image more closely resembles the original image meaning that the watermark is more imperceptible. The PSNR is defined as

$$
P S N R=10 \log _{10}\left(\frac{255}{\sqrt{M S E}}\right)^{2}
$$

where, the mean square error is defined as

$$
M S E=\frac{1}{\mathrm{M} \times \mathrm{N}} \sum_{i=1}^{\mathrm{M}} \sum_{j=1}^{\mathrm{N}}(X(i, j)-W(i, j))^{2} .
$$

where, $X(i, j)$ is a pixel of the original host or cover image of size $\mathrm{M} \times \mathrm{N}$ and $W(i, j)$ is a pixel of the watermarked image of 
size $\mathrm{M} \times \mathrm{N}$.

The robustness of the algorithm determined in terms of correlation factor. The similarity and differences between original watermark and extracted watermark is measured by Normalized correlation (NC). Its value generally 0 to 1 . Ideally it should be 1 and acceptable value is 0.7 [12].

$$
N C=\frac{\sum_{i=1}^{\mathrm{M}} \sum_{j=1}^{\mathrm{N}}\left(W_{\text {org }}(i, j) \times W_{\text {rec }}(i, j)\right)}{\sqrt{\sum_{i=1}^{\mathrm{M}} \sum_{j=1}^{\mathrm{N}} W_{\text {org }}^{2}(i, j)} \sqrt{\sum_{i=1}^{\mathrm{M}} \sum_{j=1}^{\mathrm{N}} W_{r e c}^{2}(i, j)}} .
$$

where, $W_{\text {org }}(i, j)$ is a pixel of the original watermark of size $\mathrm{M} \times \mathrm{N}$ and $W_{\text {rec }}(i, j)$ is a pixel of the recovered watermark of size $\mathrm{M} \times \mathrm{N}$.

\section{NUMERICAL EXPERIMENTS}

We implemented the proposed algorithm using MATLAB 2016a with system specifications windows 7 OS, Intel i5 core processor and 64-bit operating system. We evaluated the performance of the combined DWT-DCT-SVD based watermarking algorithm. The gray scale Barbara, Lena and peppers images of size $512 \times 512$ as host images for which embedding watermark effectively and cameraman image of size $256 \times 256$ is used as watermark image as shown in Figure 1. This section provides experimental results of proposed DWT-DCT-SVD based watermarking scheme was tested with three different images Barbara, Lena, and Peppers using various image processing attacks such as rotation attack, salt and pepper attack, Gaussian noise attack, sharpening and noise and speckle attack with the watermarked images.

The performance of the proposed algorithm of experimental results shows in Table 1 and Table 2. Obtained results (see Table 1) shows that the PSNR value between host and watermarked images are $51.30 \mathrm{~dB}$ and $71.23 \mathrm{~dB}$ at scale factor alpha $(\alpha)=0.01$ and 0.001 with $\mathrm{NC}$ value 1 and 0.999 respectively. In order to achieve better performance compared to Ganic and Eskicioglu [27], Liu and Tan [28] and Lai and Tsai [19], and NC values shown in Table 2 against Gaussian noise attack.

Table 1. Comparison of imperceptibility (PSNR) with the work of Ganic [27], Liu \&Tan [28] and Lai \& Tsai [19]

\begin{tabular}{ccc}
\hline Method & Scale factor $(\alpha)$ & PSNR \\
\hline Ganic [27] & 0.01 & 37.80 \\
Liu \& Tan [28] & 0.01 & 51.50 \\
Lai [19] & 0.01 & 51.14 \\
Proposed & 0.01 & $\mathbf{5 1 . 3 0}$ \\
& 0.001 & $\mathbf{7 1 . 2 3}$ \\
\hline
\end{tabular}

Table 2. Experimental results showing Normalized Correlation (NC)

\begin{tabular}{ccc}
\hline & \multicolumn{2}{c}{ Gaussian noise attack $($ GN) } \\
\hline Method & Scale factor & NC \\
\hline Ganic [27] & 0.01 & 0.9377 \\
Liu \& Tan [28] & 0.01 & 0.8953 \\
Lai \& Tsai [19] & 0.01 & 0.9756 \\
Proposed & 0.9 & 0.9904 \\
\hline
\end{tabular}

Table 3 shows that the results of image watermarking process without noise attacks. The Cameraman image is extracted from Barbara watermarked image with PSNR $31.6845 \mathrm{~dB}$ and $51.3307 \mathrm{~dB}$ at scale factor alpha $(\alpha)=0.1$ and 0.01 respectively and truncated singular values $k 1=253$, $k 2=250$. The PSNR calculated for Lena image are $31.7082 \mathrm{~dB}$ and $51.3016 \mathrm{~dB}$ at scale factor alpha $(\alpha)=0.1$ and 0.01 respectively, also extracted watermark from Lena watermarked image with truncated values $k 1=251, k 2=251$ at a scale factor alpha $(\alpha)=0.1$ and 0.01 .

For pepper image, PSNR obtained $31.0842 \mathrm{~dB}$ and 50.6766 $\mathrm{dB}$ at scale factor alpha $(\alpha)=0.1$ and 0.01 respectively and the watermark extracted by Pepper watermarked image with truncated values $k 1=253, k 2=250$ and $k 1=252, k 2=251$ at scale factor alpha $(\alpha)=0.1$ and 0.01 respectively.

Table 3. MSE, PSNR, NC and Truncated results for watermarked images without noise attack

\begin{tabular}{|c|c|c|c|c|c|}
\hline Images & $\begin{array}{c}\text { Scale } \\
\text { factor } \\
(\alpha) \\
\end{array}$ & MSE & $\begin{array}{l}\text { PSNR } \\
\end{array}$ & NC & $\begin{array}{c}\text { Truncated } \\
\text { values } \\
(\mathbf{k} 1, \mathbf{k} 2) \\
\end{array}$ \\
\hline \multirow[t]{3}{*}{ Barbara } & 0.1 & $1.0117 \mathrm{e}-8$ & 31.6845 & 1 & $(253,250)$ \\
\hline & 0.01 & $4.8092 \mathrm{e}-7$ & 51.3307 & 1 & $(253,250)$ \\
\hline & 0.001 & $1.7222 \mathrm{e}-4$ & 71.2573 & 0.9997 & $(253,250)$ \\
\hline \multirow[t]{3}{*}{ Lena } & 0.1 & $1.1073 \mathrm{e}-8$ & 31.7082 & 1 & $(251,251)$ \\
\hline & 0.01 & $4.5003 e-7$ & 51.3016 & 1 & $(251,251)$ \\
\hline & 0.001 & $8.499 \mathrm{e}-5$ & 71.2331 & 0.9999 & $(251,251)$ \\
\hline \multirow[t]{3}{*}{ Peppers } & 0.1 & $4.8381 \mathrm{e}-9$ & 31.0842 & 1 & $(253,250)$ \\
\hline & 0.01 & $4.5203 \mathrm{e}-7$ & 50.6766 & 1 & $(252,251)$ \\
\hline & 0.001 & $1.3819 \mathrm{e}-4$ & 70.6005 & 0.9998 & $(252,251)$ \\
\hline
\end{tabular}

Table 4. Normalized correlation (NC) and MSE for Barbara watermarked image due to three different noise ataacks at scale factor alpha $=0.9$ and tolerence $(\mathrm{t})=0.01$

\begin{tabular}{|c|c|c|c|c|c|}
\hline Image & $\begin{array}{c}\text { Noise } \\
\text { attack }\end{array}$ & Variance & $\begin{array}{c}\text { Scale } \\
\text { factor } \\
(\alpha)\end{array}$ & MSE & $\mathbf{N C}$ \\
\hline \multirow{24}{*}{ Barbara } & \multirow{8}{*}{ Gaussian } & 0.01 & 0.9 & 0.0122 & 0.9938 \\
\hline & & 0.02 & 0.9 & 0.0168 & 0.9886 \\
\hline & & 0.03 & 0.9 & 0.0217 & 0.9810 \\
\hline & & 0.04 & 0.9 & 0.0263 & 0.9735 \\
\hline & & 0.05 & 0.9 & 0.0306 & 0.9651 \\
\hline & & 0.06 & 0.9 & 0.0346 & 0.9582 \\
\hline & & 0.07 & 0.9 & 0.0385 & 0.9512 \\
\hline & & 0.08 & 0.9 & 0.0425 & 0.9426 \\
\hline & \multirow{8}{*}{$\begin{array}{l}\text { Salt and } \\
\text { pepper }\end{array}$} & 0.01 & 0.9 & 0.0078 & 0.9965 \\
\hline & & 0.02 & 0.9 & 0.0090 & 0.9949 \\
\hline & & 0.03 & 0.9 & 0.0106 & 0.9929 \\
\hline & & 0.04 & 0.9 & 0.0124 & 0.9905 \\
\hline & & 0.05 & 0.9 & 0.0140 & 0.9874 \\
\hline & & 0.06 & 0.9 & 0.0162 & 0.9840 \\
\hline & & 0.07 & 0.9 & 0.0179 & 0.9814 \\
\hline & & 0.08 & 0.9 & 0.0207 & 0.9765 \\
\hline & \multirow{8}{*}{$\begin{array}{c}\text { Noise } \\
\text { speckle }\end{array}$} & 0.01 & 0.9 & 0.0121 & 0.9952 \\
\hline & & 0.02 & 0.9 & 0.0160 & 0.9930 \\
\hline & & 0.03 & 0.9 & 0.0193 & 0.9909 \\
\hline & & 0.04 & 0.9 & 0.0230 & 0.9876 \\
\hline & & 0.05 & 0.9 & 0.0263 & 0.9849 \\
\hline & & 0.06 & 0.9 & 0.0393 & 0.9814 \\
\hline & & 0.07 & 0.9 & 0.0327 & 0.9780 \\
\hline & & 0.08 & 0.9 & 0.0360 & 0.9749 \\
\hline
\end{tabular}


Table 5. Normalized correlation (NC) and MSE for Lena watermarked image due to three different noise ataacks at scale factor alpha $=0.9$ and tolerence $(t)=0.01$

\begin{tabular}{|c|c|c|c|c|c|}
\hline Image & $\begin{array}{c}\text { Noise } \\
\text { attack }\end{array}$ & Variance & $\begin{array}{c}\text { Scale } \\
\text { factor } \\
(\alpha)\end{array}$ & MSE & $\mathrm{NC}$ \\
\hline \multirow{24}{*}{ Lena } & \multirow{8}{*}{ Gaussian } & 0.01 & 0.9 & 0.0115 & 0.9904 \\
\hline & & 0.02 & 0.9 & 0.0164 & 0.9834 \\
\hline & & 0.03 & 0.9 & 0.0212 & 0.9754 \\
\hline & & 0.04 & 0.9 & 0.0261 & 0.9671 \\
\hline & & 0.05 & 0.9 & 0.0302 & 0.9600 \\
\hline & & 0.06 & 0.9 & 0.0354 & 0.9498 \\
\hline & & 0.07 & 0.9 & 0.0398 & 0.9412 \\
\hline & & 0.08 & 0.9 & 0.0432 & 0.9345 \\
\hline & \multirow{8}{*}{$\begin{array}{l}\text { Salt and } \\
\text { pepper }\end{array}$} & 0.01 & 0.9 & 0.0073 & 0.9945 \\
\hline & & 0.02 & 0.9 & 0.0088 & 0.9924 \\
\hline & & 0.03 & 0.9 & 0.0104 & 0.9899 \\
\hline & & 0.04 & 0.9 & 0.0127 & 0.9865 \\
\hline & & 0.05 & 0.9 & 0.0145 & 0.9832 \\
\hline & & 0.06 & 0.9 & 0.0167 & 0.9795 \\
\hline & & 0.07 & 0.9 & 0.0191 & 0.9753 \\
\hline & & 0.08 & 0.9 & 0.0214 & 0.9713 \\
\hline & \multirow{8}{*}{$\begin{array}{c}\text { Noise } \\
\text { speckle }\end{array}$} & 0.01 & 0.9 & 0.0110 & 0.9924 \\
\hline & & 0.02 & 0.9 & 0.0147 & 0.9890 \\
\hline & & 0.03 & 0.9 & 0.0181 & 0.9852 \\
\hline & & 0.04 & 0.9 & 0.0214 & 0.9814 \\
\hline & & 0.05 & 0.9 & 0.0254 & 0.9769 \\
\hline & & 0.06 & 0.9 & 0.0284 & 0.9729 \\
\hline & & 0.07 & 0.9 & 0.0320 & 0.9685 \\
\hline & & 0.08 & 0.9 & 0.0361 & 0.9637 \\
\hline
\end{tabular}

Table 6. Normalized correlation (NC) and MSE for Peppers watermarked image due to three different noise ataacks at scale factor alpha $=0.9$ and tolerence $(\mathrm{t})=0.01$

\begin{tabular}{|c|c|c|c|c|c|}
\hline Image & $\begin{array}{l}\text { Noise } \\
\text { attack }\end{array}$ & Variance & $\begin{array}{c}\text { Scale } \\
\text { factor } \\
(\alpha)\end{array}$ & MSE & $\mathrm{NC}$ \\
\hline \multirow{24}{*}{ Peppers } & \multirow{8}{*}{ Gaussian } & 0.01 & 0.9 & 0.098 & 0.9920 \\
\hline & & 0.02 & 0.9 & 0.0153 & 0.9839 \\
\hline & & 0.03 & 0.9 & 0.0202 & 0.9757 \\
\hline & & 0.04 & 0.9 & 0.0251 & 0.9665 \\
\hline & & 0.05 & 0.9 & 0.0293 & 0.9596 \\
\hline & & 0.06 & 0.9 & 0.0333 & 0.9524 \\
\hline & & 0.07 & 0.9 & 0.0370 & 0.9454 \\
\hline & & 0.08 & 0.9 & 0.0409 & 0.9370 \\
\hline & \multirow{8}{*}{$\begin{array}{l}\text { Salt and } \\
\text { pepper }\end{array}$} & 0.01 & 0.9 & 0.0047 & 0.9969 \\
\hline & & 0.02 & 0.9 & 0.0062 & 0.9944 \\
\hline & & 0.03 & 0.9 & 0.0082 & 0.9916 \\
\hline & & 0.04 & 0.9 & 0.0098 & 0.9888 \\
\hline & & 0.05 & 0.9 & 0.0122 & 0.9849 \\
\hline & & 0.06 & 0.9 & 0.0144 & 0.9810 \\
\hline & & 0.07 & 0.9 & 0.0162 & 0.9776 \\
\hline & & 0.08 & 0.9 & 0.0196 & 0.9714 \\
\hline & \multirow{8}{*}{$\begin{array}{l}\text { Noise } \\
\text { speckle }\end{array}$} & 0.01 & 0.9 & 0.0093 & 0.9946 \\
\hline & & 0.02 & 0.9 & 0.0131 & 0.9912 \\
\hline & & 0.03 & 0.9 & 0.0169 & 0.9876 \\
\hline & & 0.04 & 0.9 & 0.0209 & 0.9832 \\
\hline & & 0.05 & 0.9 & 0.0242 & 0.9793 \\
\hline & & 0.06 & 0.9 & 0.0276 & 0.9748 \\
\hline & & 0.07 & 0.9 & 0.0315 & 0.9704 \\
\hline & & 0.08 & 0.9 & 0.0352 & 0.9649 \\
\hline
\end{tabular}

NC value 1 indicates extracted watermark is exactly identical to the original image as shown in Figure 2. The Barbara, Lena and Peppers watermarked images rotated by $30^{\circ}$ and extracted watermark with $\mathrm{NC}$ values $0.9542,0.9066$ and 0.9364 respectively. The watermarked images due to various kinds of attacks for Barbara, Lena and Peppers images as shown in Figure 3 to Figure 5.

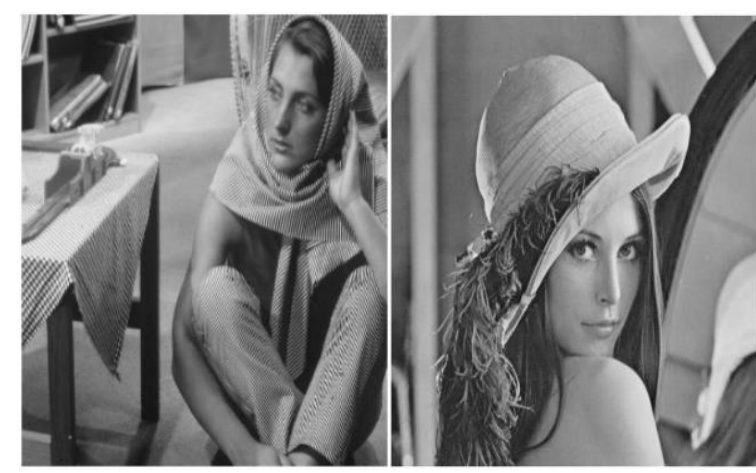

(a) Barbara

(b) Lena

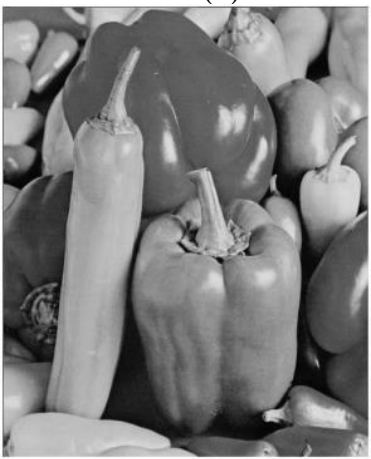

(c) Peppers

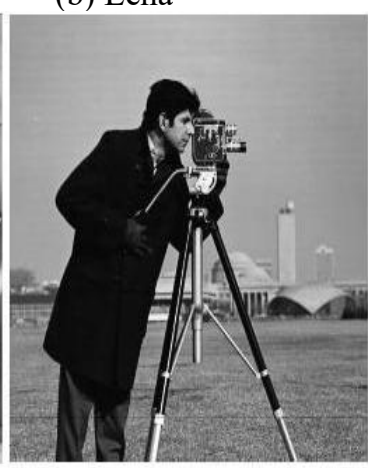

(d) Cameraman
Figure 1. Original host images (a), (b), (c) and original watermark image $(d)$

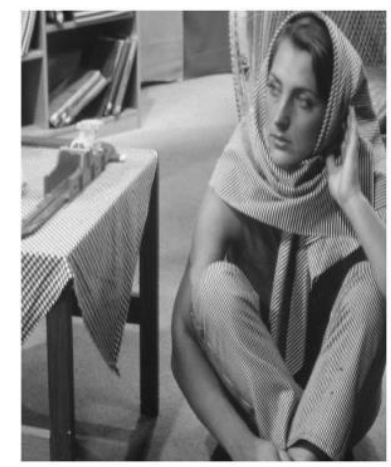

(a)

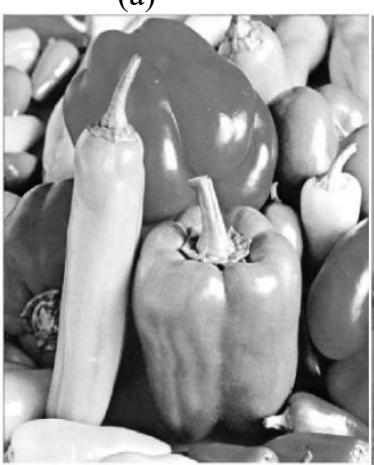

(c)

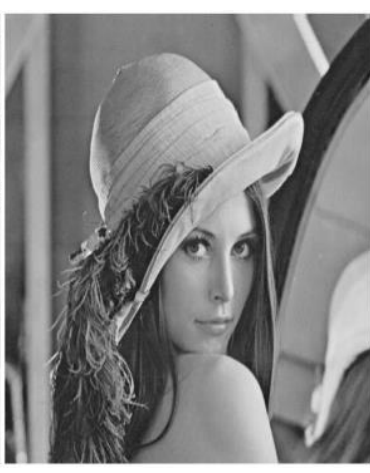

(b)

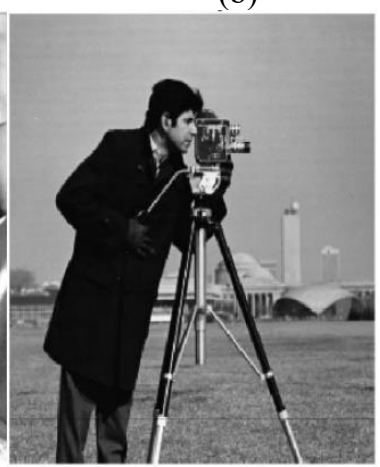

(d)

Figure 2. Watermarked images (a), (b), (c) and extracted watermark image without noise attacks (d) 
In the Table 4 to Table 6 , NC values have been evaluated for Gaussian noise, salt and pepper noise and noise speckle at noise densities from 0.01 to 0.08 . We extracted watermark images due to various kinds of attacks with $\mathrm{NC}$ values greater than 0.9 as shown in Figure 6 and obtained result demonstrate better performance at scale factor alpha $(\alpha)=0.9$. In the experiments, we used tolerance $(\mathrm{t})$ as 0.01 . However, the graphical representation of the performance by the proposed method as shown in Figure 7 to Figure 9 reveals NC values are very close to 1 against wide set of attacks. From this analysis, illustration clearly demonstrates that the proposed method is evident from simulation results are better strength and imperceptibility for attacked image.

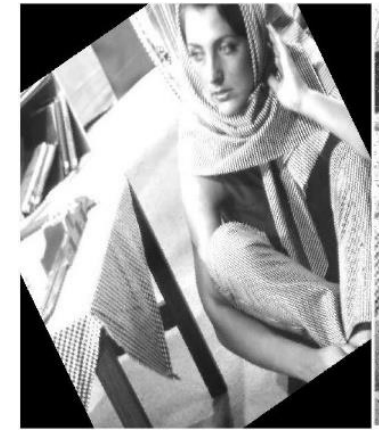

(a) Rotation attack by $30^{\circ}$

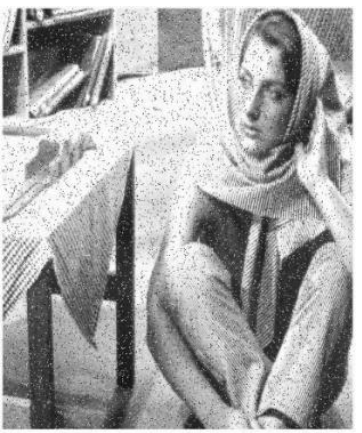

(b) Salt and pepper noise

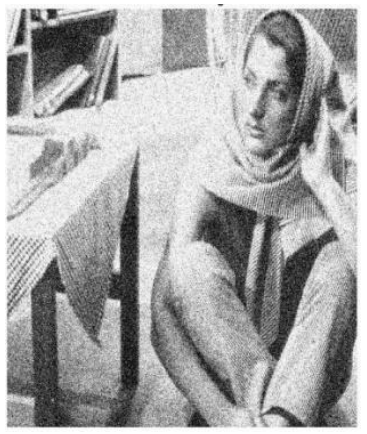

(c) Gaussian noise attack

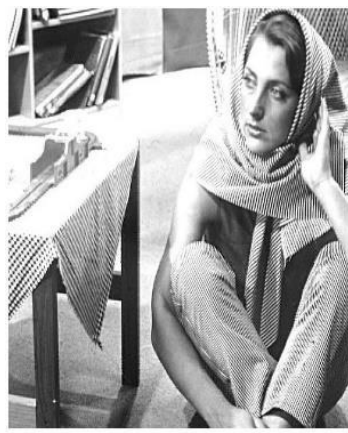

(d) Sharpening

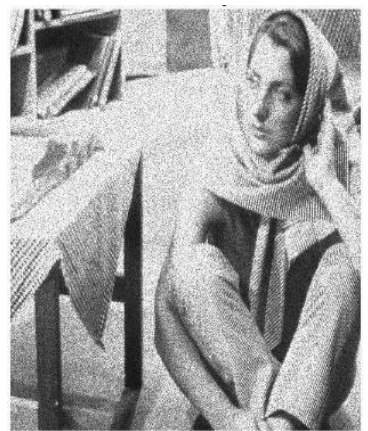

(e) Speckle noise attack

Figure 3. Watermarked Barbara images with different kinds of attacks

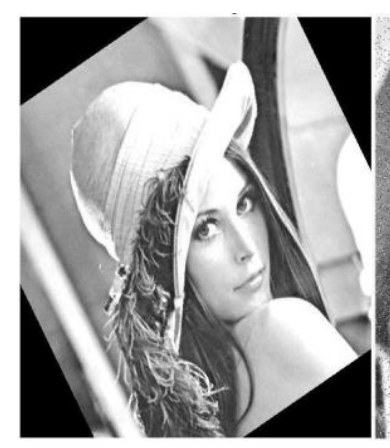

(a) Rotation attack by $30^{\circ}$

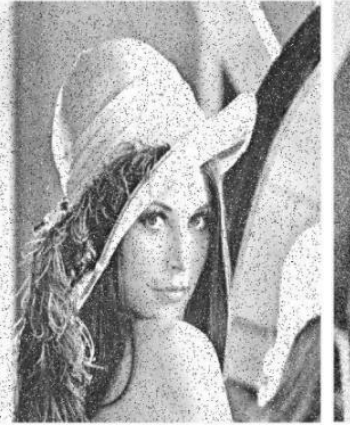

(b) Salt and pepper noise

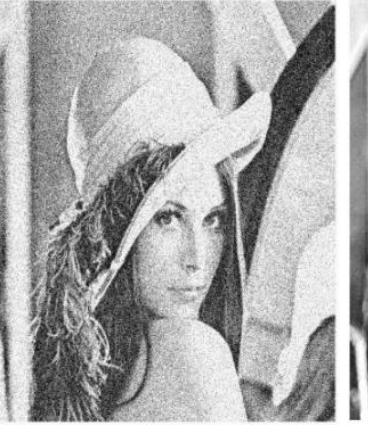

(c) Gaussian noise attack

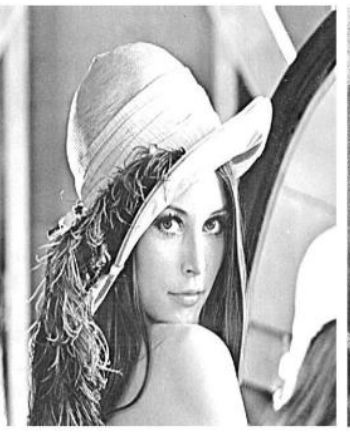

(d) Sharpening

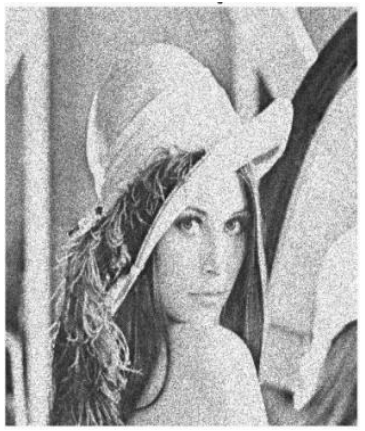

(e) Speckle noise attack

Figure 4. Watermarked Lena images with various kinds of attacks

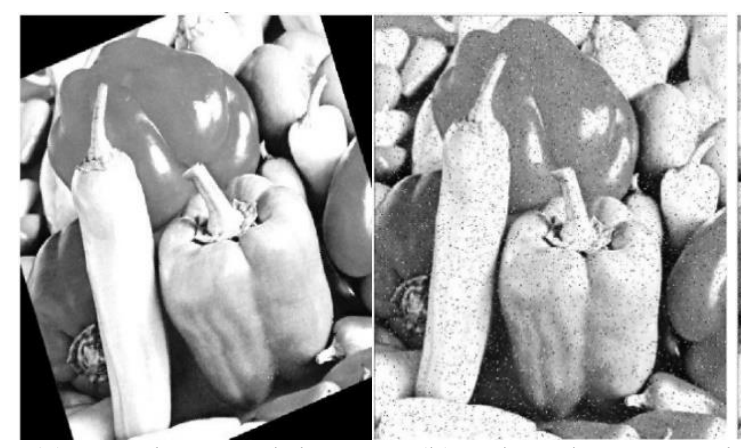

(a) Rotation attack by $30^{\circ}$ (b) Salt and pepper noise

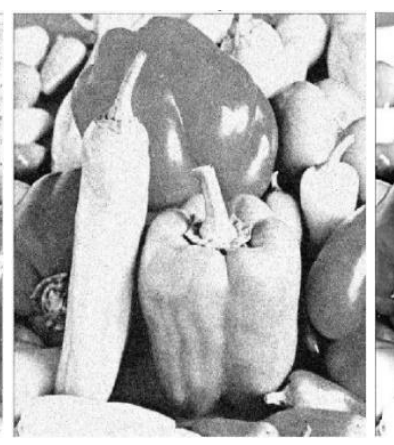

(c) Gaussian noise attack

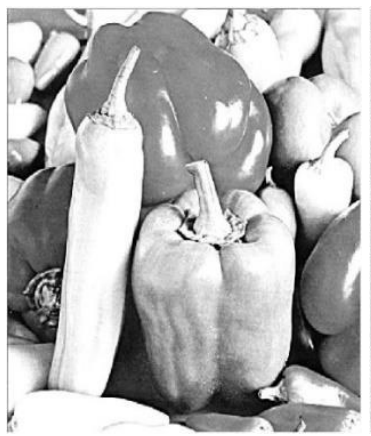

(d) Sharpening

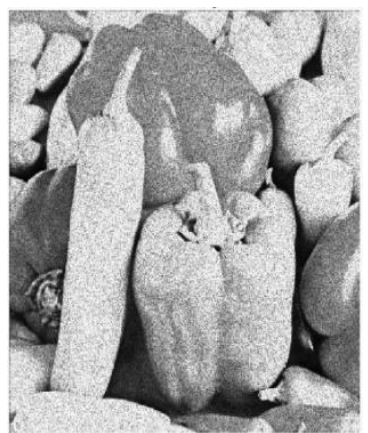

(e) Speckle noise attack

Figure 5. Watermarked Pepper images with various kinds of attacks

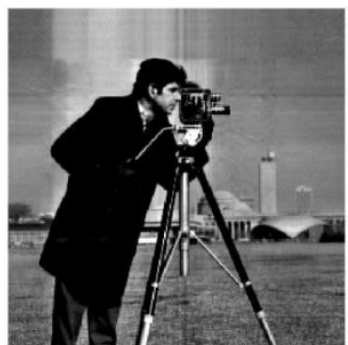

(a) By rotation attack

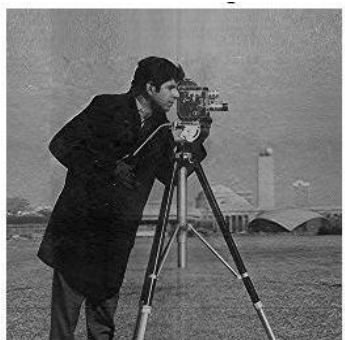

(b) By salt and pepper noise

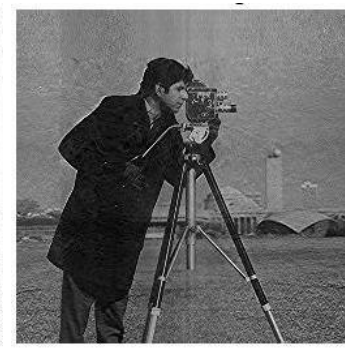

(c) By Gaussian noise

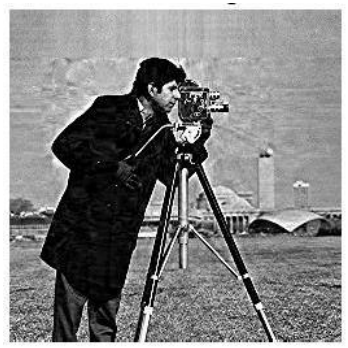

(d) By sharpening

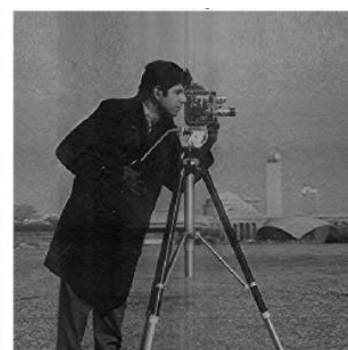

(e) By Speckle noise

Figure 6. Extracted watermark images by different kinds of attacks at alpha $=0.9$ and tolerence $(\mathrm{t})=0.01$ 


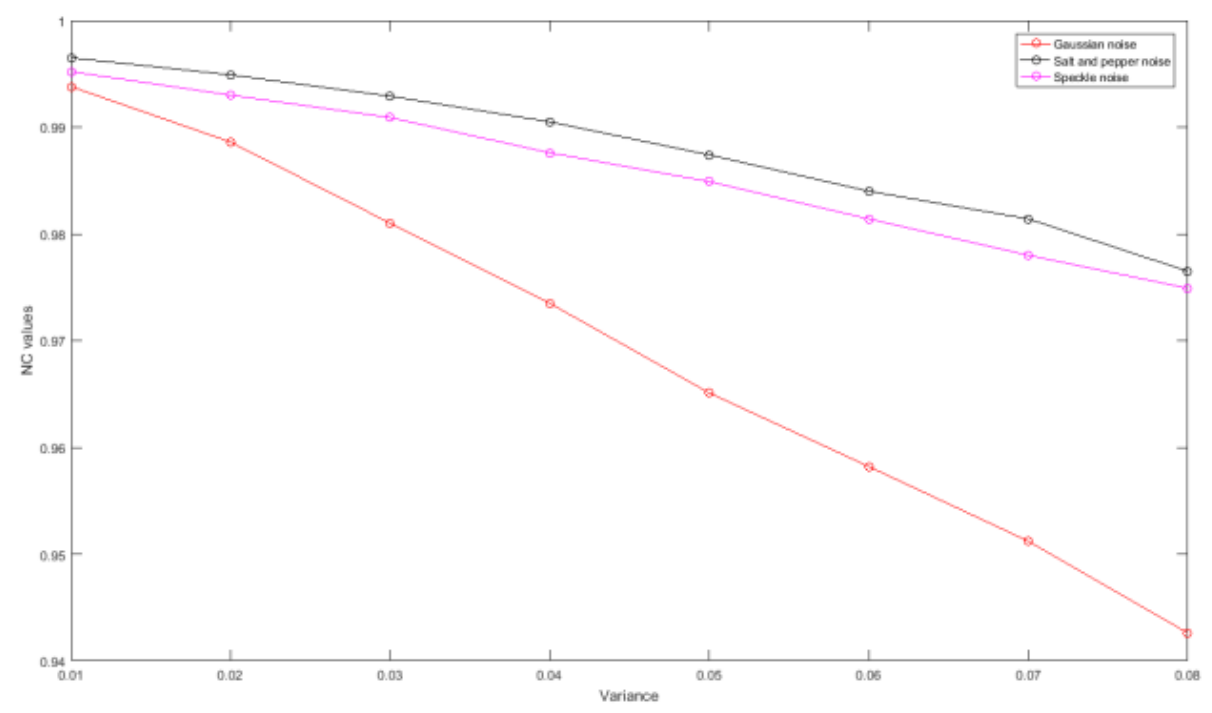

Figure 7. Performance of the proposed method showing NC values for Barbara watermarked image attacked by Gaussian noise, salt and pepper noise and speckle noise against different noise densities

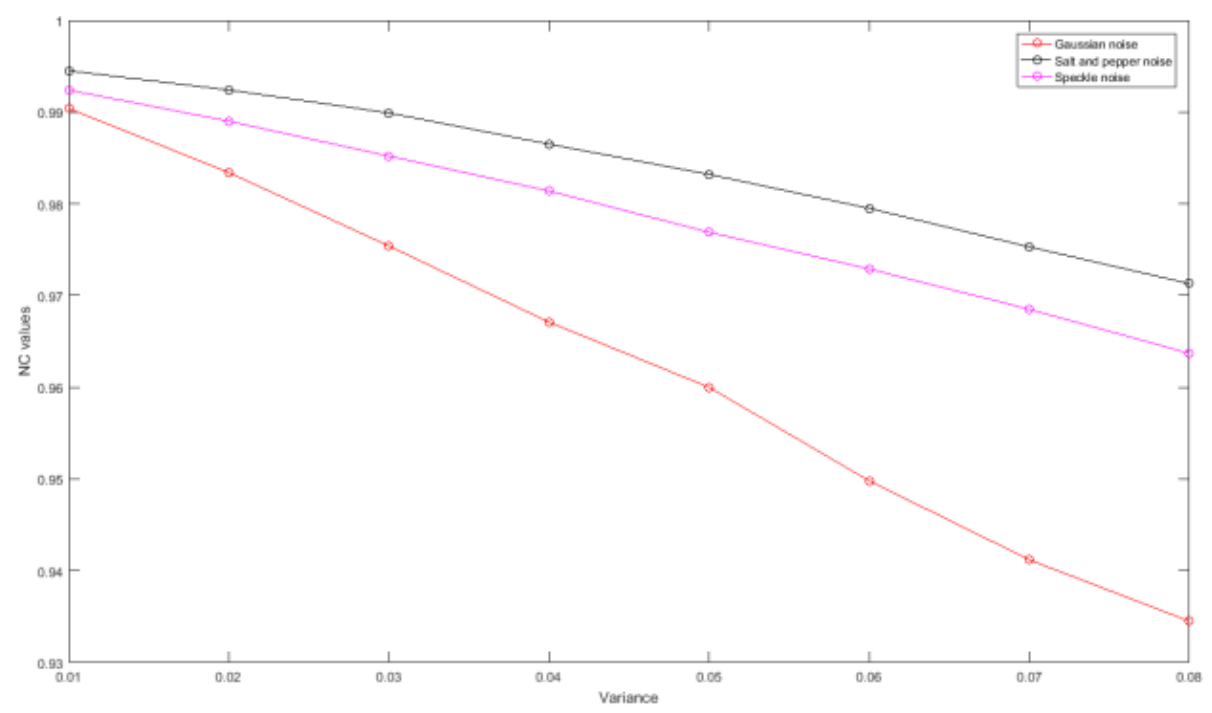

Figure 8. Performance of the proposed method showing NC values for Lena watermarked image attacked by Gaussian noise, salt and pepper noise and speckle noise against different noise densities

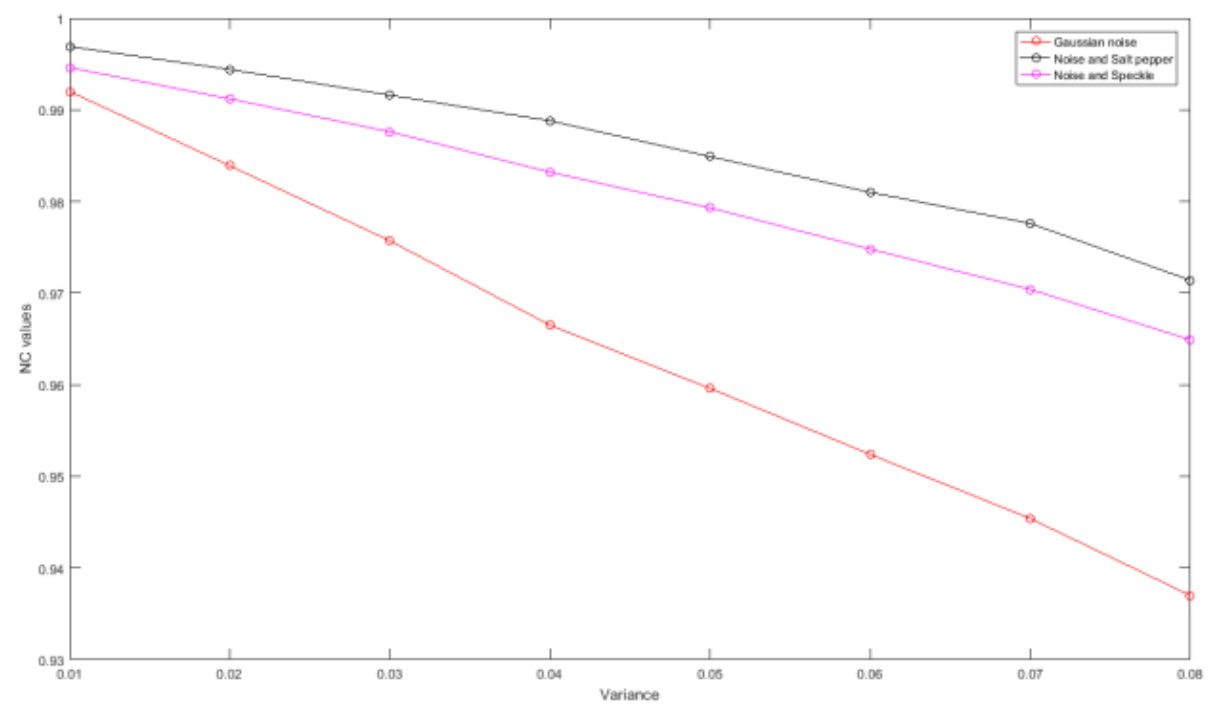

Figure 9. Performance of the proposed method showing NC values for Peppers watermarked image attacked by Gaussian noise, salt and pepper noise and speckle noise against different noise densities 


\section{CONCLUDING REMARKS}

In this paper, the proposed algorithm significant role of improvement in imperceptibility and robustness under several image processing attacks for different images. In the simulation results, some attacks such as rotation attack, salt and pepper attack, Gaussian noise attack, sharpening and speckle noise have been applied to evaluate the performance of the proposed algorithm in comparison with the other watermarking methods. Finally, advantage of the proposed algorithm to achieve best performance in robustness without losing the quality of the image. For future work proposed algorithm leads to different types of transform based methods to improvement in image watermarking and steganography.

\section{ACKNOWLEDGMENTS}

Author Riyajuddin is thankful to Govt. of Karnataka Directorate of Minorities (GOKDOM), India for providing minority fellowship to carry out the present research work.

\section{REFERENCES}

[1] Danyali, H., Makhloghi, M., Tab, F.A. (2011). Robust blind DWT based digital image watermarking using singular value decomposition. International Journal of Innovative Computing, Information and Control, 8(7): 4691-4703.

[2] Piva, A., Barni, M., Bartolini, F. (1998). Copyright protection of digital images by means of frequency domain watermarking. Proc. of the SPIE Conference on Mathematics of Data/Image Coding, Compression, and Encryption, USA. https://doi.org/10.1117/12.330369

[3] Wolfgang, R.B., Podilchuk, C.I., Delp, E.J. (1999). Perceptual watermarks for digital images and video. Proceedings of the IEEE, 87(7): 1108-1126.

[4] Naveenakumara, U., Reddy, A.P. (2020). On the image watermarking of compressed image samples using Singular Value Thresholding in the wavelet domain. Global Journal of Pure and Applied Mathematics, 16(3): 495-507.

[5] Khan, A., Mirza, A.M. (2007) Genetic perceptual shaping: utilizing cover image and conceivable attack information during watermark embedding. International Journal of Information Fusion, 8(4): 354-365. https://doi.org/10.1016/j.inffus.2005.09.007

[6] ElShafie, D.R., Kharma, N., Ward, R. (2008) Parameter optimization of an embedded watermark using a genetic algorithm. 2008 3rd International Symposium on Communications, Control and Signal Processing, pp. 1263-1267.

https://doi.org/10.1109/ISCCSP.2008.4537420

[7] Amiri, S.H., Jamzad, M.A. (2009). A robust image watermarking method in wavelet domain using genetic algorithm. 2009 International Conference on Availability, Reliability and Security, pp. 612-617. https://doi.org/10.1109/ARES.2009.123

[8] Singh, A., Tayal, A. (2012). Choice of wavelet from wavelet families for DWT-DCT-SVD image watermarking. International Journal of Computer Applications, 48(17): 9-14 https://doi.org/10.5120/7439-0375
[9] Singh, A.K., Dave, M., Mohan, A. (2013). A hybrid algorithm for image watermarking against signal processing attacks. In: Ramanna S., Lingras P., Sombattheera C., Krishna A. (eds) Multi-disciplinary Trends in Artificial Intelligence. MIWAI 2013. Lecture Notes in Computer Science, vol 8271. Springer, Berlin, Heidelberg. https://doi.org/10.1007/978-3-642-449499_22

[10] Navas, K., Ajay, M.C., Lekshmi, M., Archana, T.S., Sasikumar, M. (2008). DWT-DCT-SVD based watermarking. 2008 3rd International Conference on Communication Systems Software and Middleware and Workshops (COMSWARE '08), pp. 271-274. https://doi.org/10.1109/COMSWA.2008.4554423

[11] Wang, B., Ding, J., Wen, Q., Liao, X., Liu, C. (2008). An image watermarking algorithm based on DWT DCT and SVD. 2009 IEEE International Conference on Network Infrastructure and Digital Content, pp. 1034-1038. https://doi.org/10.1109/ICNIDC.2009.5360866

[12] Khan, M.I., Rahman, M., Sarker, M., Hasan, I. (2013). Digital watermarking for image authentication based on combined DCT, DWT and SVD transformation. International Journal of Computer Science Issues (IJCSI), 10(3): 223-230.

[13] Harish, N., Kumar, B., Kusagur, A. (2013). Hybrid robust watermarking techniques based on DWT, DCT, and SVD. International Journal of Advanced Electrical and Electronics Engineering (IJAEEE), 2(5): 137-143.

[14] Shaikh, H., Khan, M.I., Kelkar, Y. (2013). Analysis of robustness of hybrid digital image watermarking technique under various attacks. International Journal of Computer Science and Mobile Computing, 2(3): 137-143.

[15] Madhesiya, S., Ahmed, S. (2013). Advanced technique of digital watermarking based on SVD-DWT-DCT and Arnold transform. International Journal of advanced Research in Computer Engineering \& Technology (IJARCET), 2(5): 1918-1923.

[16] Golshan, F., Mahammadi, K. (2013). A hybrid intelligent SVD-based perceptual shaping of a digital image watermark in DCT and DWT domain. The Imaging Science Journal, 61(1): 35-46. https://doi.org/10.1179/1743131X11Y.0000000049

[17] Srivastava, A., Saxena, P. ((2013). DWT-DCT-SVD based semi blind image watermarking using middle frequency band. IOSR Journal of Computer Engineering, 12(2): 63-66.

[18] Mei, J., Li, S., Tan, X. (2009). A digital watermarking algorithm based on DCT and DWT. Proceedings of the 2009 International Symposium on Web Information Systems and Applications (WISA'09), pp. 104-107.

[19] Lai, C.C., Tsai, C.C. (2010). Digital image watermarking using discrete wavelet transform and singular value decomposition. IEEE Transactions on Instrumentation and Measurement, 59(11): 3060-3063. https://doi.org/10.1109/TIM.2010.2066770

[20] Ruch, D.K., Van Fleet, P.J. (2011). Wavelet Theory: An Elementary Approach with Applications. John Wiley \& Sons.

[21] Hong, W., Hang, M. (2006). Robust digital watermarking scheme for copy right protection. IEEE Trans. Signal Process, 2(1): 1-8.

[22] Mallat, S.G. (1989). A theory for multiresolution signal decomposition: The wavelet representation. IEEE Transactions on Pattern Analysis and Machine 
Intelligence,

11(7):

https://doi.org/10.1109/34.192463

[23] Meerwald, P., Uhl, A. (2001). Survey of wavelet-domain watermarking algorithms. Security and Watermarking of Multimedia Contents III, pp. 505-516. https://doi.org/10.1117/12.435434

[24] Rao, K.R., Yip, P. (2014). Discrete Cosine Transform, Algorithms, Advantages, Applications. Academic Press.

[25] Golub, G.H., Van Loan, C.F. (2013). Matrix Computations. (Vol. 3), JHU Press.

[26] Hansen, P.C., Nagy, J.G., O’Leary, D.P. (2006).
Deblurring Images Matrices, Spectra and Filtering. SIAM.

[27] Ganic, E., Eskicioglu, A.M. (2004). Robust DWT-SVD domain image watermarking: Embedding data in all frequencies. Proc. Workshop Multimedia Security, Magdeburg, Germany, pp. 166-174. https://doi.org/10.1145/1022431.1022461

[28] Liu, R., Tan, T. (2002). An SVD-based watermarking scheme for protecting rightful ownership. IEEE Transactions on Multimedia, 4(1): 121-128. https://doi.org/10.1109/6046.985560 